\title{
Evaluation of Salusin $\beta$ in Patients with Prediabetes and Type 2 Diabetes Mellitus
}

\author{
Sabah Hussein dayekh ${ }^{1}$, Mohammed Imran Hamzah ${ }^{1}$, Jelal Abid Ateaai ${ }^{2}$ \\ ${ }^{1}$ Department of Chemistry and Biochemistry, College of Medicine/AL-Nahrain University, Iraq, \\ ${ }^{2}$ Department of Internal Medicine / College of Medicine, AL- Nahrain University, Iraq
}

\begin{abstract}
Background: Type 2 Diabetes mellitus is a group of metabolic disease characterized by increase blood glucose level (hyperglycemia) resulting from either defect in insulin production by pancreas or increase insulin resistance that lead to decrease insulin effect in the body. prediabetes It is stage before diabetes mellitus including impaired fasting glucose (IFG)where the fasting glucose level $(6.1-7.0 \mathrm{mmol} / \mathrm{L})$ and impaired glucose tolerance (IGT after glucose load) glucose level $(7.8-11 \mathrm{mmol} / \mathrm{L})$.(salusin- $\boldsymbol{\beta}$ ) It is a newly glass of peptide it is synthesized in many body tissues, like (skeletal muscle, human vascular smooth muscle cells, and endothelial muscle) it have important role in Diabetes pathogenesis it has been reported to involve in vascular inflammation and the relation between salusin $\beta$ and diabetes through endothelial injury, salusin $\beta$ increase inflammation in diabetes. It have essential role in pathological endothelial dysfunction. Method: by using ELISA kit, we measured serum level of salusin $\beta$ in 30 patients with prediabetes and 30 patients with type 2 DM compare with 30 healthy volunteers enlisted as normal controls. Result: serum salusin $\beta$ level in patient with type $2 \mathrm{DM}$ were significantly higher than prediabetes group and normal subjects. Salusin $\beta$ in prediabetes group no significant higher than in healthy control subjects. Conclusion: Serum Salusin $\beta$ level in patients with type 2 DM are expressed at significantly high levels were no difference in Salusin $\beta$ level of prediabetes group and normal control subjects.
\end{abstract}

Keywords: Salusin $\beta$, diabetes mellitus, prediabetes, ELISA.

\section{Introduction}

Diabetes mellitus is a group of metabolic disease characterized by increase blood glucose level (hyperglycemia) resulting from either defect in insulin production by pancreas or increase insulin resistance that lead to decrease insulin effect in the body. Feature of hyperglycemia include polyuria, weight loss, polyphagia, polydipsia, unclear .

Prdiabetes It is stage before diabetes mellitus including impaired fasting glucose (IFG)where the fasting glucose level (6.1-7.O mmol/L) and impaired glucose tolerance (IGT after glucose load) glucose level $(7.8-11 \mathrm{mmol} / \mathrm{L})$, to forms of prediabetes in which increase glucose level more than normal range but below the renal threshold of diabetes level, the HbA1c range in the two. state. The presence of prediabetes is associated with the presence of $\beta$ cell dysfunction and insulin resistance and about $5-10 \%$ of population are development to. Salusin $\beta$ It is a newly glass of . salusin- $\beta$ have special physicochemical properties, It is present in biological fluids such as human plasma, urine and vascular .the bioactive peptide (salusin- $\beta$ ) is synthesized in many body tissues, stomach, bone marrow, small intestine, thymus, lymph, salivary glands, adrenal medulla, adrenal cortex, spleen, liver, brain, skeletal muscle, testes, human vascular smooth muscle cells, and endothelial muscle. salusin $\beta$ it have important role in the diabetes. it has been reported to involve in vascular inflammation and the relation between salusin $\beta$ and diabetes through endothelial injury, salusin $\beta$ increase oxidative stress, cardiac dysfunction and inflammation in diabetes. It have essential role in pathological endothelial.

The aim of the present study was to evaluate serum level of Salusin $\beta$ in patients with type $2 \mathrm{DM}$, Prediabetes, and compare this group with control healthy group as well as to investigate whether it can be used as 
a biomarkers for detection of diabetes.

\section{Materials and Method}

\section{Study design case control study}

The present study include 90 Iraqi participants (30 with type $2 \mathrm{DM}, 30$ with prediabetes, 30 normal healthy control group) the Age range (30-65)years, the age and gender matched to the patients and control group. Blood sample collected between September 2018 and april 2019. The following biochemical parameters have been studied. Salusin $\beta$, hs- CRP by ELISA method, fasting blood sugar (FBS), HbA1c, blood urea, serum creatinine, Lipid profile by colorimetric method by (cobas C111). Measure body mass index (BMI), and measure (WC). Fasting serum Samples were collected from patients and control by taking eight (8) $\mathrm{ml}$ of venous blood will be collected in plane tube (without anticoagulant). Blood are stand for 30 minutes then centrifuged in 1800 RPM for 15 minutes. Serums are transferred to new tube and keep in $-20 \mathrm{C}$ until assay.

\section{Salusin ELISA}

A total $100 \mu \mathrm{l}$ serum was analyzed using a commercially available ELISA kit Salusin $\beta$ (melsin) according to manufactures recommendations. Prepare all re a $\mathrm{g}$ e $\mathrm{n} \mathrm{t}$ s before starting assay procedure. It is recommended that all Standards and Samples be added in duplicate to the Microelisa Stripplate. Add standard: Set Standard wells, testing sample wells. Add standards $50 \mu 1$ to standard wells. Add $10 \mu 1$ of testing sample of sample wall .Then add $40 \mu$ l of sample diluent to testing sample well, doesn't add anything to the Blank well. Add HRP-conjugate reagent $100 \mu 1$ to each well, covering with an adhesive strip, incubate for 60 minutes at $37^{\circ} \mathrm{C}$. Aspirate each well and wash, repeating the wash 4 times for a total of 5 washes. Wash by filling each well with Wash Solution $(400 \mu l)$ using a squirt bottle, manifold dispenser or auto washer. Removal of liquid completely at each step is essential to good results. After the last wash, remove any remaining Wash Solution by decanting or aspirating. Invert the plate and blot it on a clean paper towels. Add $50 \mu \mathrm{l}$ of chromogen solution (A) and added $50 \mu 1$ of chromogen solution B to each well. mix Gently and incubate for fifty minutes (15) minutes at $37^{\circ} \mathrm{C}$. and Protect from light. Add $50 \mu \mathrm{l}$ from Stop Solution to each well. The color should change from blue to yellow of the walls. If the color in the wells is green or the color change does not appear uniform, gently tap the plate to ensure thorough mixing. Read the Optical Density (O.D.) at wave lengths $450 \mathrm{~nm}$ and using a microtiter plate reader within 15 minutes.

\section{Statistical Analysis}

Statistical analysis was carried out by using SPSS version 20 and Microsoft excel 2013.the numerical data expressed as mean $\pm \mathrm{SD}$. Comparison between mean serum Salusin $\beta$ concentration and (IFG, DM) groups were performed. Receiver Operating Characteristics (ROC) Curve was calculated to estimate the sensitivity and specificity of the used Salusin $\beta$.

\section{Result}

There was highly significant difference in mean Salusin- $\beta$ among study groups $(P<0.001)$; the level was highest in diabetic patients with highly significant difference $(P<0.001)$ in comparison with that of both prediabetics and control subjects; however, there was insignificant difference between control and prediabetic groups $(P=0.736)$,

Table 1. Salusin- $\beta$ in patients and control groups

\begin{tabular}{|c|c|c|c|c|c|c|c|c|c|c|}
\hline \multirow{2}{*}{ Characteristic } & \multicolumn{2}{|c|}{ Control } & \multicolumn{2}{|c|}{ Prediabetic } & \multicolumn{2}{|c|}{ Diabetic } & \multirow{2}{*}{$\mathbf{P}$} & \multirow{2}{*}{ P1 } & \multirow{2}{*}{$\mathbf{P 2}$} & \multirow{2}{*}{ P3 } \\
\hline & Mean & SD & Mean & SD & Mean & SD & & & & \\
\hline Salusin- $\beta(\mathrm{pg} / \mathrm{ml})$ & 5.12 & 1.15 & 5.27 & 0.83 & 7.80 & 2.47 & $\begin{array}{l}<0.001 \\
\dagger \\
\mathrm{HS}\end{array}$ & $\begin{array}{l}0.736 \\
¥ \\
\text { NS }\end{array}$ & $\begin{array}{l}<0.001 \\
¥ \\
\text { HS }\end{array}$ & $\begin{array}{l}<0.001 \\
¥ \\
\text { HS }\end{array}$ \\
\hline
\end{tabular}

$n$ : number of cases; data were presented as either mean and standard deviation; $\uparrow$ : One way ANOVA; ¥: post hoc LSD test; HS: highly significant at $P \leq 0.01$; NS: not significant at $P \leq 0.05$; S: significant at $P \leq 0.05$; $P 1$ : Control vs prediabetic; $P 2$ : Control vs DM; $P 3$ : Prediabetic vs DM 


\section{Result}

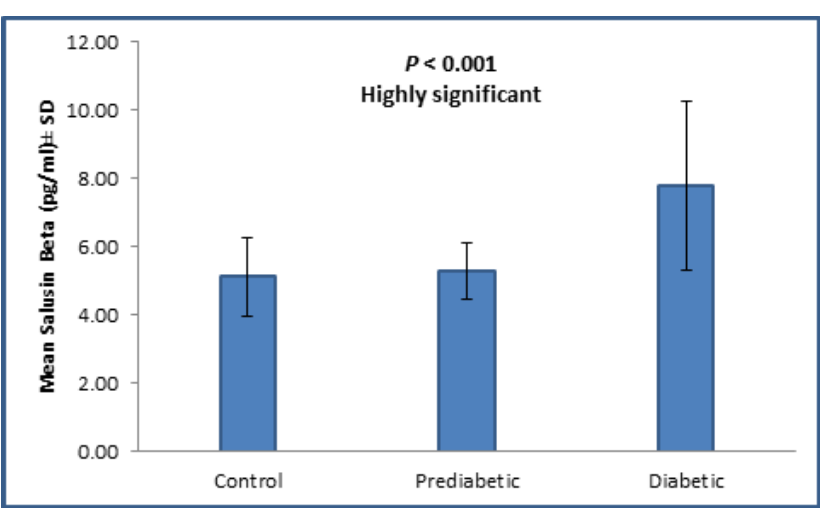

Figure 1. Bar chart showing the mean serum salusin beta in diabetics, prediabetics and control subjects

Because of the highly significant difference in the level of salusin- $\beta$ in diabetic patients when compared to prediabetics and control groups, the authors of the current study suggested the presence of a cutoff value that can predict a diagnosis of diabetes with certain level of accuracy. For that reason, receiver operator characteristic (ROC) curve analysis was carried out and the results are shown in figure 3.1 and table 3.6. The cute off value was $>6.5$ with an accuracy level of $88.2 \%$, a sensitivity level of $80 \%$ and specificity level of $93.3 \%$, table 3.6.

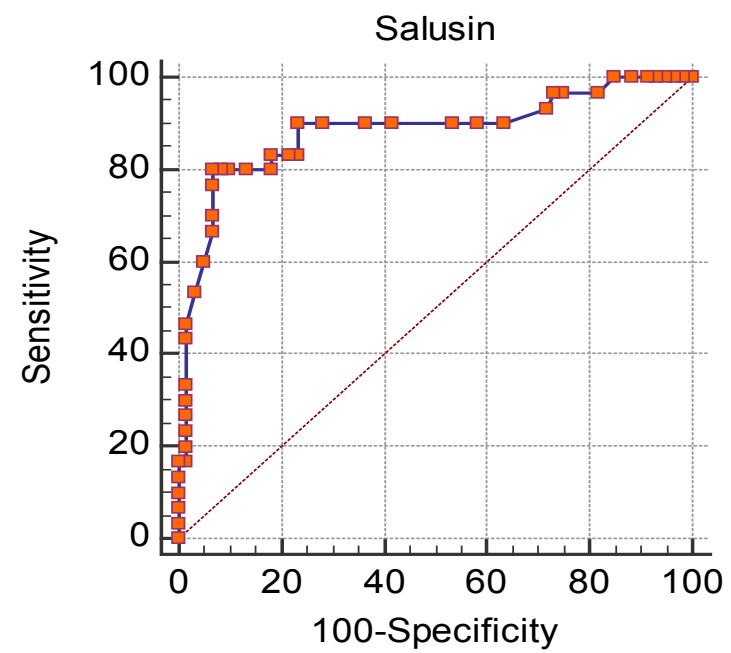

Figure 2. Receiver operator characteristic (ROC) curve to predict the Salusin $\beta$ cutoff value with a diagnosis of diabetes mellitus
Table 2. Cutoff value, sensitivity and specificity of Salusin- $\beta$.

\begin{tabular}{|l|l|}
\hline Characteristic & Salusin- $\boldsymbol{\beta}$ \\
\hline Cutoff value & $>6.5$ \\
\hline $\begin{array}{l}\text { AUC } \\
(95 \% \text { CI })\end{array}$ & $\begin{array}{l}0.882 \\
(0.796-0.940)\end{array}$ \\
\hline Accuracy & $88.2 \%$ \\
\hline P & $<0.001$ \\
\hline Sensitivity & 80.00 \\
\hline Specificity & 93.33 \\
\hline
\end{tabular}

\section{Discussion}

. It is a peptide that present in endothelium tissue and in cell membrane it is play important role in endothelial function and pathogenesis of diabetes the increase level of salusin $\beta$ in patients with type -2 diabetes mellitus related with the complication of diabetes due to endothelial injury (7). In the present study found increase in Salusin $\beta$ concentration in diabetes group and high significant deference between diabetes group and another two groups at $\mathrm{p}$ value $(<0.001)$ and normal level of prediabetes and control group. And this agreement with study done by (7) salusin $\beta$ was higher in level with high glucose (diabetes group). Salusin beta was positivelycorrelated to female gender There was no significant correlation among markers in all three study groups, as shown in table (3.3) Because of the highly significant difference in the level of salusin- $\beta$ in diabetic patients when compared to prediabetics and control groups, the authors of the current study suggested the presence of a cutoff value that can predict a diagnosis of diabetes with certain level of accuracy. For that reason, receiver operator characteristic (ROC) curve analysis was carried out and The cute off value was $>6.5$ with an accuracy level of $88.2 \%$, sensitivity level of $80 \%$ and specificity level of $93.3 \%$, result show in table (3.6). Salusin beta was negatively correlated to age, positively correlated to FBS, positively correlated to HBA1c, negatively correlated to cholesterol and negatively correlated to LDL, in the present study the result show there was positive correlation between salusin- $\beta$ and FBS. And no correlation between salusin- $\beta$ and adiponectin, no correlation with lipid bound sialic acid and no correlation with lipid profile. 
Table 3. Correlations of biochemical markers to demographic, disease characteristic lipid profile and renal function in prediabetic group

\begin{tabular}{|l|l|l|}
\hline \multirow{2}{*}{ Characteristic } & \multicolumn{2}{|l|}{ salusin $(\mathbf{p g} / \mathbf{m l})$} \\
\cline { 2 - 3 } & $\boldsymbol{r}$ & $\boldsymbol{P}$ \\
\hline Age & 0.312 & 0.094 \\
\hline Gender & 0.449 & $0.013 *$ \\
\hline BMI & 0.171 & 0.365 \\
\hline Waste $(\mathrm{cm})$ & -0.134 & 0.479 \\
\hline FBS & 0.103 & 0.587 \\
\hline HbAlc & 0.025 & 0.896 \\
\hline cholesterol (mg/dl) & -0.194 & 0.305 \\
\hline TG (mg/dl) & -0.206 & 0.276 \\
\hline HDL $(\mathrm{mg} / \mathrm{dl})$ & 0.048 & 0.801 \\
\hline LDL (mg/dl) & -0.312 & 0.093 \\
\hline VLDL (mg/dl) & -0.205 & 0.278 \\
\hline UREA (mg/dl) & -0.122 & 0.52 \\
\hline creatinine (mg/dl) & 0.223 & 0.237 \\
\hline
\end{tabular}

* significant at $P \leq 0.05$; ** highly significant at $P \leq 0.01$

\section{Conclusion}

Salusin $\boldsymbol{\beta}$ was significant increase level in patients with type -2 diabetes mellitus as a compared with prediabetes and control healthy group with high sensitive and Specific of cutoff value for diagnosis type $2 \mathrm{DM}$, there was significant correlation between salusin $\beta$ and FBS and HbA1c. no significant deference of salusin- $\beta$ level in prediabetes and control groups.

Ethical Clearance: The Research Ethical Committee at scientific research by ethical approval of both environmental and health and higher education and scientific research ministries in Iraq

Conflict of Interest: The authors declare that they have no conflict of interest.

Funding: Self-funding

\section{References}

1. Association AD.Diagnosis and classification of diabetes mellitus. Diabetes care. 2012;35(1) S64-S71.

2. American Diabetes Association. Classification and diagnosis of diabetes. Sec.Standards of Medical Care in Diabetes.2016;39(1):S13-S22.

3. Bansal, N. Prediabetes diagnosis and treatment A review . World journal of diabetes.2015;(6):296.

4. Burns C, \& Sirisena I. Type 2 Diabetes: Etiology, Epidemiology Pathogenesis, Treatment. Metabolic Syndrome: A Comprehensive Textbook 2016;601617.

5. Osei K, Gaillard T, Schuser D. Plasma adiponectin levels in high risk African-Americans with normal glucose tolerance, impaired glucose tolerance, and type 2 diabetes. Obesity Research;2005;13:179-85.

6. Wtanabe T, Nishio K, Kanome T, Mastuyama TA, Koba S, Sakai T. Impact of salusin -alpha and beta on human macrophage foam cell formation and coronary atherosclerosis. 2008;117(5):638-48.

7. Zhu X, Zhou W, Cai, Sun H, and Qiu L. Salusin $\beta$ mediates -high glucose-induced endothelial injury via disruption of AMPK signaling pathway, biochemical and biophysical Research Communications, 2018;491(2):515-521.

8. Citil C, Koner V, Aydin S, Yilmaz M, Albayrak S, Ozercan IH. Brain, Liver and serum salusin alpha and salusin beta alterations in Sprague-Dawley rats with or without metabolic syndrome. Med Sci Moint, 2014;(20):1326-33. 